\title{
Premature ovarian failure risk factors in an Iranian population
}

This article was published in the following Dove Press journal:

International Journal of General Medicine

12 April 2012

Number of times this article has been viewed

\author{
Alieh Ghassemzadeh ${ }^{1,2}$ \\ Laya Farzadi ${ }^{1,2}$ \\ Elaheh Beyhaghi, \\ 'Women's Reproductive Health \\ Research Center, Tabriz University of \\ Medical Sciences, Tabriz, Iran; ${ }^{2}$ Alzahra \\ University Hospital, Department of \\ Obstetrics and Gynecology, Tabriz \\ University of Medical Sciences, \\ Tabriz, Iran
}

Background: The aim of this study was to determine possible correlates of premature ovarian failure (POF) in an Iranian population.

Methods: In a case-control study, 80 patients with POF were compared with 80 controls enrolled from the same setting during 2007-2008. A food diary was used to assess food consumption habits.

Results: Mean age of starting ovarian failure symptoms was $19.3 \pm 5.7$ years and mean age of menopause was $22.6 \pm 6.3$ years. Familial coincidence was observed in 16 POF patients versus no one in the control group $(P<0.05)$. POF patients had lower frequency of both eating red meat and fish when compared with controls $(P<0.001)$. POF and control subjects consumed similar amounts of dairy products, being $5.3 \pm 3.2$ times per week in POF and 5.6 \pm 2.1 times in the control groups.

Conclusion: In this study, an association between POF and lower red meat or fish consumption was found.

Keywords: POF, etiology, case-control, nutrition, premature menopause, premature ovarian dysfunction, primary ovarian insufficiency

\section{Introduction}

Premature ovarian failure (POF) is the occurrence of amenorrhea in conjunction with raised serum follicle stimulating hormone (FSH $>40 \mathrm{IU} / \mathrm{L})$ before the age of 40 years. ${ }^{1}$ Terminology is inconsistent. Some other terms have been used such as, premature menopause, premature ovarian dysfunction, and primary ovarian insufficiency. It is reported to occur in $0.9 \%-3.0 \%$ of the general female population. ${ }^{2}$ For every decade before the age of 40, the prevalence of POF is estimated to decrease by a factor of 10 . POF is the etiology in $10 \%-28 \%$ of the cases with primary amenorrhea and in $4 \%-18 \%$ of those with secondary amenorrhea. ${ }^{3} \mathrm{POF}$, other than affecting different aspects of quality of life, may lead to infertility, osteoporosis, and cardiovascular disorders, which puts it on high priority of reproductive and health research. ${ }^{4}$ Turner syndrome and gonadal dysgenesis are the best known causes of early POF. Nevertheless, in normal 46XX karyotype females presenting with early POF, the etiology is most often unknown. ${ }^{3}$ There are ethnic differences in prevalence of POF in different populations. ${ }^{5}$ The ethnic prevalence variations pose possible etiologic differences also due to environmental, genetic, and nutritional factors. Conduction of etiologic studies in different settings or populations can help in improving the knowledge regarding etiology and predictors of POF. The aim of this study was to determine possible correlates of POF in an Iranian population.
Correspondence: Laya Farzadi Alzahra University Hospital, Artesh Ave, Tabriz, Iran Tel +984II 32919130 Fax +98 4II 5566449 Email l.farzadi@yahoo.com submit your manuscript | www.dovepress.com

Dovepress 


\section{Methods}

In a case-control study, 80 patients with POF were compared with 80 controls enrolled during 2007-2008. Study setting was Alzahra University Hospital and Tabriz Subspecialty Clinic for Infertility. Cases were menopause women under the age of 40 , referred to the study centers. All the cases had referred due to amenorrhea and infertility and were assessed two times for serum FSH; they were assessed for POF if FSH was found to be above $30 \mathrm{U} / \mathrm{L}$. Eighty patients lacking the outcome of interest were also enrolled as controls.

Food consumption variables were measured using a food diary assessment tool, measuring average frequency of consumed food. Controls were selected from other clinics of Alzahra University Hospital. They were matched with cases for age using a frequency matching technique.

Data were entered into the computer and analyzed using bivariate statistical tests as well as multivariate regression analysis. All the test results were interpreted as two-tailed results, and a $P$ value $<0.05$ was considered as statistically significant.

The study protocol was approved by the regional committee of ethics in Tabriz University of Medical Sciences.

\section{Results}

Mean (and standard deviation) of the age of the patients were 30.3 (5.9) and 30 (5.6) years, respectively, for the POF and control groups $(P=0.3)$. Eighty-five percent of POF and $76 \%$ of control group subjects had urban residence $(P=0.2)$. Education level distribution compared between groups was not statistically significant (Figure 1).

Mean age of starting ovarian failure symptoms was $19.3 \pm 5.7$ years, and mean age of menopause was $22.6 \pm 6.3$ years. In 26 patients in this group, menstruations continued dependent on drug treatment. Maternal menopausal age, if reached, was $45.0 \pm 3.8$ years in the POF group

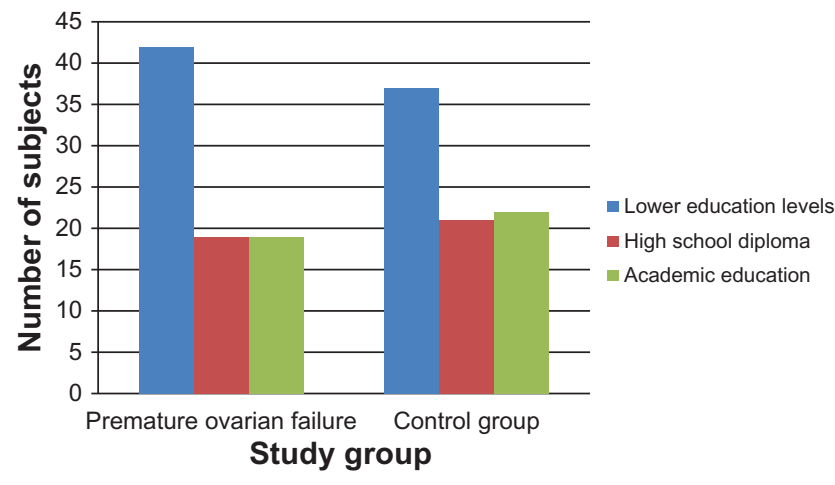

Figure I Distribution of education level compared between primary ovarian failure group and control group subjects. versus $46.6 \pm 3.3$ years in the control group $(P<0.05)$. Mean age of menarche was $13.9 \pm 2.0$ years for the POF group and $13.6 \pm 1.2$ years in the control group. The difference was not statistically significant. A history of lifelong irregular menstruation was reported in $76.3 \%$ of participants in the POF group, while no one reported an irregular menstruation history in the control group $(P<0.001)$. Seven patients in the POF group had a history of ovarian surgery. The surgery type was cystectomies in five patients and cauterization in two cases. Mumps history was reported in 16 POF versus 25 control participants. The difference was not statistically significant. Familial coincidence was observed in 16 POF patients versus no one in the control group $(P<0.05)$. Familial marriage of the parents was the case in 23 and 30 subjects in POF and controls, respectively. The difference was not found to be statistically significant. None of the patients in either group had a history of radiotherapy or chemotherapy. Only four patients in the POF group reported history of renal disease $(P=0.1)$. Diabetes, thyroidal disease, lupus erythematous, and rheumatoid arthritis didn't exist in any of the participants of the study groups.

POF patients had lower frequency of consuming both red meat and fish compared with controls $(P<0.001)$. POF patients had a mean 2.8 times of red meat consumption and 0.23 times of fish consumption per week. Controls had a mean 4.6 times of red meat consumption and 1.37 times of fish consumption per week. Vegetable consumption as reported by the POF patients had a mean of $3.1 \pm 2.2$ times per week. Mean weekly consumption of vegetables was recorded to be $3.5 \pm 3.3$ times per week among controls. The difference was not statistically different. POF patients consumed mean 0.4 times of fast foods per week. The figure was 0.5 times per week for controls $(P=0.3)$. POF and control subjects consumed similar amounts of dairy products, being $5.3 \pm 3.2$ times per week in the POF group and 5.6 \pm 2.1 times in the control group $(P=0.5)$. Also, living close to high voltage power posts, living close to industrial centers, and using mobile phones were not found to be associated with POF ( $P$ values were $0.210,0.444$, and 0.200 , respectively). None of the participants were smokers.

Using multivariate regression analysis, the amount of weekly consumption of red meat and fish appeared as independent predictors of POF.

\section{Discussion}

Some of the possible predictors of POF were not found significant. This is possibly due to lower incidence and compared variability between groups. For example, contrary 
to many foreign studies, smoking is rare among women in this context and there were no smokers in either group, so the study could not investigate an association between smoking and POF. No doubt, in cases where there is a lack of variation as in the present study, the factors will be classed as insignificant. Nevertheless, smoking is repeatedly reported to be associated with POF. ${ }^{6-10}$ In the present study, a history of irregular menstruation was found to be strongly associated with POF. This was in line with a large Italian study reporting that the risk of POF and of early menopause was higher among women reporting lifelong irregular menstrual cycles. ${ }^{11}$ The present study was a case-control study, and the Italian study was cross-sectional study; however, due to lack of clear-cut temporality assessment, in both cases, caution needs to be taken in interpreting the results. Considering this point and also lack of a reasonable biological plausibility to explain a direct causality association, it cannot be clarified with certainty whether irregular menstrual cycles can be considered as a risk factor, a predisposing factor, or even an early symptom of POF. ${ }^{12}$ Nevertheless, some studies have also supported lack of an association between irregular menstrual cycles and ovarian failure. ${ }^{13}$ Due to such an uncertainty, the authors of the present paper preferred to assess and discuss the association just through a bivariate analysis rather than including it in multivariate analysis.

There was no association found between age at menarche and POF that was consistent with the results of the Italian study, but controversial results can also be retrieved from literature. ${ }^{11,14,15}$ As in the present study, insufficient or no cases of ovarian surgery, chemotherapy, or radiotherapy existed to investigate existence of association between these factors and POF. However, such an association seems quite plausible and is also reported in previous studies. ${ }^{16-18}$ Although the present study was not supported to do genetic assessments, family clustering of disease was a significant finding. Although preferential recall of family history by women with early menopause could contribute to the association between family history and early menopause observed in this study, a genetic factor is also plausible, including partial deletions of the X chromosome, compatible with the deficiency of male siblings in cases with family history of early menopause. ${ }^{19}$ A hereditary or genetic link to POF is well discussed and substantially supported in previous POF literature. ${ }^{20-24}$

Interestingly, in the present study there was an association between POF and lower red meat or fish consumption. If we consider this to be a possible causal effect, the mechanism may be sought in dietary amino acid balance, minerals such as zinc in all of kinds of meat, omega fatty acids in fish, and other potential mechanisms. However, a major confounder in this regard is the economic level, ${ }^{25,26}$ which is very difficult to measure and control for in developing countries and is recommended to be considered in future research.

\section{Disclosure}

The authors report no conflicts of interest in this work.

\section{References}

1. Coulam CB, Adamson SC, Annegers JF. Incidence of premature ovarian failure. Obstet Gynecol. 1986;67(4):604-606.

2. Falsetti L, Scalchi S, Villani MT, Bugari G. Premature ovarian failure. Gynecol Endocrinol. 1999;13(3):189-195.

3. Kokcu A. Premature ovarian failure from current perspective. Gynecol Endocrinol. 2010;26(8):555-562.

4. Anasti JN. Premature ovarian failure: an update. Fertil Steril. 1998;70(1): $1-15$.

5. Nippita TA, Baber RJ. Premature ovarian failure: a review. Climacteric. 2007;10(1):11-22.

6. Luoto R, Kaprio J, Uutela A. Age at natural menopause and sociodemographic status in Finland. Am J Epidemiol. 1994;139(1):64-76.

7. Cramer DW, Harlow BL, Xu H, Fraer C, Barbieri R. Cross-sectional and case-controlled analyses of the association between smoking and early menopause. Maturitas. 1995;22(2):79-87.

8. Nilsson P, Moller L, Koster A, Hollnagel H. Social and biological predictors of early menopause: a model for premature aging. J Intern Med. 1997;242(4):299-305.

9. Harlow BL, Signorello LB. Factors associated with early menopause. Maturitas. 2000;35(1):3-9.

10. Cooper GS, Sandler DP, Bohlig M. Active and passive smoking and the occurrence of natural menopause. Epidemiology. 1999;10(6): 771-773.

11. Premature ovarian failure: frequency and risk factors among women attending a network of menopause clinics in Italy. BJOG. 2003; 110(1):59-63.

12. Chang SH, Kim CS, Lee KS, et al. Premenopausal factors influencing premature ovarian failure and early menopause. Maturitas. 2007;58(1): 19-30.

13. Testa G, Chiaffarino F, Vegetti W, et al. Case-control study on risk factors for premature ovarian failure. Gynecol Obstet Invest. 2001; 51(1):40-43.

14. Parazzini F, Negri E, La VC. Reproductive and general lifestyle determinants of age at menopause. Maturitas. 1992;15(2):141-149.

15. Bromberger JT, Matthews KA, Kuller LH, Wing RR, Meilahn EN, Plantinga P. Prospective study of the determinants of age at menopause. Am J Epidemiol. 1997;145(2):124-133.

16. Weinstein LS, Liu J, Sakamoto A, Xie T, Chen M. Minireview: GNAS: normal and abnormal functions. Endocrinology. 2004;145(12): 5459-5464.

17. Hoek A, Schoemaker J, Drexhage HA. Premature ovarian failure and ovarian autoimmunity. Endocr Rev. 1997;18(1):107-134.

18. Santoro N. Research on the mechanisms of premature ovarian failure. J Soc Gynecol Investig. 2001;8(1 Suppl):S10-S12.

19. Cramer DW, Xu H, Harlow BL. Family history as a predictor of early menopause. Fertil Steril. 1995;64(4):740-745.

20. Rizzolio F, Bione S, Sala C, et al. Chromosomal rearrangements in Xq and premature ovarian failure: mapping of 25 new cases and review of the literature. Hum Reprod. 2006;21(6):1477-1483.

21. Prueitt RL, Ross JL, Zinn AR. Physical mapping of nine Xq translocation breakpoints and identification of XPNPEP2 as a premature ovarian failure candidate gene. Cytogenet Cell Genet. 2000;89(1-2):44-50.

22. Davison RM, Fox M, Conway GS. Mapping of the POF1 locus and identification of putative genes for premature ovarian failure. Mol Hum Reprod. 2000;6(4):314-318. 
23. Goswami D, Conway GS. Premature ovarian failure. Horm Res. 2007; 68(4):196-202.

24. Cordts EB, Christofolini DM, Dos Santos AA, Bianco B, Barbosa CP. Genetic aspects of premature ovarian failure: a literature review. Arch Gynecol Obstet. 2011;283(3):635-643.

25. Torgerson DJ, Avenell A, Russell IT, Reid DM. Factors associated with onset of menopause in women aged 45-49. Maturitas. 1994;19(2): $83-92$.
26. van Noord PA, Dubas JS, Dorland M, Boersma H, te Velde E. Age at natural menopause in a population-based screening cohort: the role of menarche, fecundity, and lifestyle factors. Fertil Steril. 1997;68(1):95-102.

\section{Publish your work in this journal}

The International Journal of General Medicine is an international, peer-reviewed open-access journal that focuses on general and internal medicine, pathogenesis, epidemiology, diagnosis, monitoring and treatment protocols. The journal is characterized by the rapid reporting of reviews, original research and clinical studies across all disease areas.
A key focus is the elucidation of disease processes and management protocols resulting in improved outcomes for the patient.The manuscript management system is completely online and includes a very quick and fair peer-review system. Visit http://www.dovepress.com/ testimonials.php to read real quotes from published authors.

Submit your manuscript here: http://www.dovepress.com/international-journal-of-general-medicine-journal 